\title{
Uniform Lipschitz Bound for a Competition Diffusion Advection System with Strong Competition
}

\author{
Tingwei Huang, Shan Zhang* \\ Department of Applied Mathematics, Nanjing University of Finance \& Economics, Nanjing, China \\ Email: *shanzhang86@163.com
}

How to cite this paper: Huang, T.W. and Zhang, S. (2021) Uniform Lipschitz Bound for a Competition Diffusion Advection System with Strong Competition. Applied Mathematics, 12, 383-398.

https://doi.org/10.4236/am.2021.125027

Received: March 22, 2021

Accepted: May 16, 2021

Published: May 19, 2021

Copyright (อ 2021 by author(s) and Scientific Research Publishing Inc. This work is licensed under the Creative Commons Attribution International License (CC BY 4.0).

http://creativecommons.org/licenses/by/4.0/

\begin{abstract}
We prove the uniform Lipschitz bound of solutions for a nonlinear elliptic system modeling the steady state of populations that compete in a heterogeneous environment. This extends known quasi-optimal regularity results and covers the optimal case for this problem. The proof relies upon the blow-up technique and the almost monotonicity formula by Caffarelli, Jerison and Kenig.
\end{abstract}

\section{Keywords}

Diffusion-Advection System, Free Boundary Problem, Uniform Lipschitz Bound

\section{Introduction}

In this paper, we consider the following competition-diffusion-advection system

$$
\begin{cases}u_{t}-\nabla \cdot\left[\mu_{1} \nabla u-\alpha u \nabla m\right]=(m-b u) u-k u v & \text { in } \Omega \times(0, \infty), \\ v_{t}-\nabla \cdot\left[\mu_{2} \nabla v-\beta v \nabla m\right]=(m-c v) v-k u v & \text { in } \Omega \times(0, \infty), \\ \mu_{1} \frac{\partial u}{\partial v}-\alpha u \frac{\partial m}{\partial v}=\mu_{2} \frac{\partial v}{\partial v}-\beta v \frac{\partial m}{\partial v}=0 & \text { on } \partial \Omega \times(0, \infty),\end{cases}
$$

where $u=u(x, t)$ and $v=v(x, t)$ denote the densities of two competing species at position $x \in \Omega$ and time $t \geq 0 . m=m(x)$ represents a local population growth rate that depends on location. In some sense, $m(x)$ can reflect the quality and quantity of resources available at the location $x$, where the favorable region $\{x \in \Omega: m(x)>0\}$ acts as a source and the unfavorable part $\{x \in \Omega: m(x)<0\}$ is a sink region [1]. $\mu_{1} \nabla u$ and $\mu_{2} \nabla v$ account for random diffusion, and $\alpha u \nabla m$ and $\beta v \nabla m$ represent movement upward along the environmental gradient. The 
two non-negative constants $\alpha$ and $\beta$ measure the tendency of the two species to move up along the gradient of $m(x)$, and $\mu_{1}$ and $\mu_{2}$ represent the random diffusion rates of two species, respectively. The positive constants $b$ and $c$ are the intraspecific and $k$ the interspecific competition rates. $\Omega$ is a bounded domain in $\mathbb{R}^{N}(N \geq 1)$ with smooth boundary $\partial \Omega$. The Zero-flux boundary condition in (1) means that no individuals cross the boundary of the habitat.

From the mathematical viewpoint, qualitative properties of non-negative solutions of system (1) have been extensively studied. We will briefly review some of them, for a more complete and detailed discussion, see [2]. For the case when $k=1, \alpha>0, \beta \equiv 0$, Cantrell et al. [3] [4] showed that if $\Omega$ is convex and $\mu_{1}=\mu_{2}$, then for positive small $\alpha$ the semi-trivial steady state $(\theta(x, \alpha, \mu), 0)$ of (1) is globally asymptotically stable. In contrast, Cantrell et al. [3] and Chen et al. [5] proved that for large values $\alpha$ system (1) can have a stable positive steady state and two competing species coexist for large $\alpha$. For the case when $k=1, \alpha>0, \beta>0$, Chen et al. [1] showed that if the ratio $\beta / \mu_{2}$ is suitable related, then the two species coexist for sufficiently large $\alpha$.

For the case when $k$ is sufficiently large, we proved in [6] that system is expected to approach a limiting configuration where all the populations survive but have disjoint habitats. Precisely, we proved that $k$-dependent solutions $\left\{\left(u_{k}, v_{k}\right)\right\}$ of (1) are uniformly bounded in Hölder spaces and they converge to the positive and negative parts of a solution of a scalar limit problem. The objective of this paper is to improve the result in [6], proving the uniform bound in Lipschitz norm. Without loss of generality, we set $\mu_{1}=\mu_{2}=1$ in system (1), and consider the time-independent case:

$$
\begin{cases}-\nabla \cdot\left[\nabla u_{k}-\alpha u_{k} \nabla m\right]=\left(m-b u_{k}\right) u_{k}-k u_{k} v_{k} & \text { in } \Omega, \\ -\nabla \cdot\left[\nabla v_{k}-\beta v_{k} \nabla m\right]=\left(m-c v_{k}\right) v_{k}-k u_{k} v_{k} & \text { in } \Omega, \\ \frac{\partial u_{k}}{\partial v}-\alpha u_{k} \frac{\partial m}{\partial v}=\frac{\partial v_{k}}{\partial v}-\beta v_{k} \frac{\partial m}{\partial v}=0 & \text { on } \partial \Omega .\end{cases}
$$

Throughout this paper, we assume that the function $m(x) \in C^{2}(\bar{\Omega})$ and $m(x)$ is positive somewhere in $\Omega$. Our main result is as follows.

Theorem 1. Let $\left\{\left(u_{k}, v_{k}\right)\right\}$ be non-negative solutions of (2). Then for every compact set $\Omega^{\prime} \subset \subset \Omega$ there exist $M>0$ independent of $k$ such that

$$
\left\|\left(u_{k}, v_{k}\right)\right\|_{L i p\left(\Omega^{\prime}\right)}:=\left\|\left(u_{k}, v_{k}\right)\right\|_{L^{\infty}\left(\Omega^{\prime}\right)}+\left\|\nabla\left(u_{k}, v_{k}\right)\right\|_{L^{\infty}\left(\Omega^{\prime}\right)} \leq M .
$$

Note that the study of strong-competition limits in corresponding elliptic or parabolic systems is of interest not only for questions of spatial segregation and coexistence, in population dynamics, as here and in [7]-[14], but also is key to the understanding of phase separation of Gross-Pitaevskii systems of modeling Bose-Einstein condensates, see [15]-[24] and reference therein.

The uniform Hölder regularity in related problems have been studied by many authors, see [10] [11] [15] [25], for the elliptic case, [15] [18] for the parabolic case, and [26] [27] [28] for the fractional diffusion case. Concerning the uniform Lipschitz boundedness, some results have already been observed in li- 
terature. For the case of two components without advection and reaction terms, Conti, Terracini and Verzini in [10] proved that if $\left\{\left(u_{k}, v_{k}\right)\right\} \in H^{1}(\Omega)$ are nonnegative solutions of

$$
\begin{cases}\Delta u_{k}=k u_{k} v_{k} & \text { in } \Omega, \\ \Delta v_{k}=\gamma k u_{k} v_{k} & \text { in } \Omega, \\ u_{k}=\phi, v_{k}=\psi & \text { on } \partial \Omega\end{cases}
$$

with $\gamma>0$ and traces $\phi, \psi \in \operatorname{Lip}(\partial \Omega)$, then $\left\{\left(u_{k}, v_{k}\right)\right\}$ is uniformly bounded in the Lipschitz norm. By using Kato's inequality, Wang and Zhang [14] generalized the result to arbitrary number of components (possibly with suitable reaction terms). In [29] Berestycki, Lin, Wei and Zhao deal with the Gross-Pitaevskii system in dimension $N=1$, they proved that if $\left\{\left(u_{k}, v_{k}\right)\right\} \in H_{0}^{1}([0,1])$ are uniformly $L^{\infty}$ bounded solutions of

$$
\begin{cases}-u_{k}^{\prime \prime}+\lambda_{1, k} u_{k}=\omega_{1} u_{k}^{3}-k u_{k} v_{k}^{2} & \text { in }[0,1] \\ -v_{k}^{\prime \prime}+\lambda_{2, k} u_{k}=\omega_{2} v_{k}^{3}-k v_{k} u_{k}^{2} & \text { in }[0,1]\end{cases}
$$

with uniformly bounded coefficients $\lambda_{i, k}, i=1,2$, then $u_{k}$ and $v_{k}$ are uniformly bounded in the Lipschitz norm. In the recent paper [30], Soave and Zilio extended the result of [10] [14] [29] to the case of arbitrary number of components and general reaction terms. The approach here follows the mainstream of [30], based upon the blow-up technique and the almost monotonicity formula by Caffarelli-Jerison-Kenig.

The rest of the paper is organized as follows: Section 2 is devoted to giving some prior estimates. Section 3 deals with the blow-up analysis. In Section 4, we prove the uniform bound in the Lipschitz norm.

\section{Some Preliminary Results}

In this section, we will derive some basic estimates. As in [1] [4], if we let $\tilde{u}_{k}=\mathrm{e}^{-\alpha m} u_{k}, \quad \tilde{v}_{k}=\mathrm{e}^{-\beta m} v_{k}$ then system (2) is equivalent to

$$
\begin{cases}-\Delta \tilde{u}_{k}=\alpha \nabla \tilde{u}_{k} \cdot \nabla m+\left(m-b \mathrm{e}^{\alpha m} \tilde{u}_{k}\right) \tilde{u}_{k}-k \mathrm{e}^{\beta m} \tilde{u}_{k} \tilde{v}_{k} & \text { in } \Omega, \\ -\Delta \tilde{v}_{k}=\beta \nabla \tilde{v}_{k} \cdot \nabla m+\left(m-c \mathrm{e}^{\beta m} \tilde{v}_{k}\right) \tilde{v}_{k}-k \mathrm{e}^{\alpha m} \tilde{u}_{k} \tilde{v}_{k} & \text { in } \Omega, \\ \frac{\partial \tilde{u}_{k}}{\partial v}=\frac{\partial \tilde{v}_{k}}{\partial v}=0 & \text { on } \partial \Omega .\end{cases}
$$

We start with the following observation of system (3).

Lemma 2. Let $\tilde{u}_{k}=\mathrm{e}^{-\alpha m} u_{k}, \tilde{v}_{k}=\mathrm{e}^{-\beta m} v_{k}$ and suppose that $\left(u_{k}, v_{k}\right)$ is a non-negative solution of (2). Then for all $x \in \bar{\Omega}$,

$$
0 \leq \tilde{u}_{k}(x) \leq \max _{\bar{\Omega}}\left\{m \mathrm{e}^{-\alpha m}\right\} / b, 0 \leq \tilde{v}_{k}(x) \leq \max _{\bar{\Omega}}\left\{m \mathrm{e}^{-\beta m}\right\} / c .
$$

Furthermore,

$$
0 \leq u_{k}(x) \leq \max _{\bar{\Omega}}\left\{\frac{m}{b}\right\} \mathrm{e}^{\alpha\left(\max _{\bar{\Omega}} m-\min _{\bar{\Omega}} m\right)}, v_{k}(x) \leq \max _{\bar{\Omega}}\left\{\frac{m}{c}\right\} \mathrm{e}^{\beta\left(\max _{\bar{\Omega}} m-\min _{\bar{\Omega}} m\right)} .
$$


Proof. We prove the estimate for $\tilde{u}_{k}$ and $u_{k}$; that for $\tilde{v}_{k}$ and $v_{k}$ follows similarly. Let $x_{0} \in \bar{\Omega}$ denote a point where $\tilde{u}_{k}\left(x_{0}\right)=\max _{\bar{\Omega}} \tilde{u}_{k}$. Assume by contradiction that

$$
\tilde{u}_{k}\left(x_{0}\right)>\max _{\bar{\Omega}}\left\{m \mathrm{e}^{-\alpha m}\right\} / b .
$$

Since $\frac{\partial \tilde{u}_{k}}{\partial v}=0$, then by the Hopf lemma $x_{0} \notin \partial \Omega$. Hence, we have $x_{0} \in \Omega$, $\nabla \tilde{u}_{k}\left(x_{0}\right)=\overrightarrow{0}$ and $\Delta \tilde{u}_{k}\left(x_{0}\right) \leq 0$. It then follows that

$$
\begin{aligned}
0 & \leq k \mathrm{e}^{\beta m\left(x_{0}\right)} \tilde{u}_{k}\left(x_{0}\right) \tilde{v}_{k}\left(x_{0}\right)-\Delta \tilde{u}_{k}\left(x_{0}\right) \\
& =\alpha \nabla \tilde{u}_{k}\left(x_{0}\right) \cdot \nabla m\left(x_{0}\right)+\left(m\left(x_{0}\right)-b \mathrm{e}^{\alpha m\left(x_{0}\right)} \tilde{u}_{k}\left(x_{0}\right)\right) \tilde{u}_{k}\left(x_{0}\right) \\
& \leq\left(\max _{\bar{\Omega}}\left\{m \mathrm{e}^{-\alpha m}\right\} / b-\tilde{u}_{k}\left(x_{0}\right)\right) b \mathrm{e}^{\alpha m\left(x_{0}\right)} \tilde{u}_{k}\left(x_{0}\right)<0 .
\end{aligned}
$$

which is a contradiction. Hence, for all $x \in \bar{\Omega}$,

$$
\tilde{u}_{k}(x) \leq \max _{\bar{\Omega}}\left\{m \mathrm{e}^{-\alpha m}\right\} / b .
$$

and for all $x \in \bar{\Omega}$,

$$
u_{k}(x)=\mathrm{e}^{\alpha m(x)} \tilde{u}_{k}(x) \leq\left(\max _{\bar{\Omega}} m / b\right) \mathrm{e}^{\alpha\left(\max _{\bar{\Omega}} m-\min _{\bar{\Omega}} m\right)} .
$$

This completes the proof of Lemma 2.

In the blow up procedure, we need the following lemma, which extends the result in [11], Lemma 4.4.

Lemma 3. Let $B_{2 R}=\left\{x \in \mathbb{R}^{N}:|x|^{2}<2 R\right\}$ be the open ball in $\mathbb{R}^{N}$. Assume that $u \in H^{1}\left(B_{2 R}\right)$ satisfying

$$
\begin{cases}-\Delta u \leq \theta \nabla m \cdot \nabla u-H u & \text { in } B_{2 R}, \\ u \geq 0 & \text { in } B_{2 R}, \\ u \leq A & \text { on } \partial B_{2 R},\end{cases}
$$

where $m \in C^{1}\left(\bar{B}_{2 R}\right)$ and $\theta, H$ are two positive constant. Then for every $\delta \in(0,1)$,

$$
\|u\|_{L^{\infty}\left(B_{R}\right)} \leq C A \mathrm{e}^{-\delta R \sqrt{H}},
$$

where $C$ is a positive constant depending only on $\delta, R$ and $\|m\|_{C^{1}\left(\bar{B}_{2 R}\right)}$.

Proof. The proof is inspired by Conti et al. [11]. Let $\sigma=\|m\|_{C^{1}\left(\bar{B}_{2 R}\right)}$ and consider the following problem:

$$
\left\{\begin{array}{l}
\varphi^{\prime \prime}(r)+\frac{N-1}{r} \varphi^{\prime}(r)=-\theta \sigma \varphi^{\prime}+H \varphi, \\
\varphi(0)=\lambda>0, \\
\varphi^{\prime}(0)=0 .
\end{array}\right.
$$

We claim that:
1) $\varphi^{\prime}(r)>0$ for $r \in(0, \infty)$;
2) $\varphi(r) \leq \lambda \mathrm{e}^{r \sqrt{H}}$ for $r \in[0, \infty)$;
3) $\varphi(r) \geq \frac{\lambda \mathrm{e}^{\theta \sigma\left(r_{0}-r\right)}}{2}\left(\frac{r_{0}}{r}\right)^{N-1} \mathrm{e}^{\left(r-r_{0}\right) \sqrt{H}}$ for $r \in\left[r_{0}, \infty\right)$, where $r_{0}>0$. 
To prove (1), we observe that $\varphi$ is defined on $[0, \infty)$ and that $\varphi>0$, $\varphi^{\prime}>0$ on $(0, \infty)$. Indeed, if not, $\varphi$ is positive on $[0, R)$ and $\varphi(R)=0$, then $\varphi^{\prime}(R) \leq 0$; On the other hand, since

$$
\left(\mathrm{e}^{\theta \sigma r} r^{N-1} \varphi^{\prime}\right)^{\prime}=\theta \sigma \mathrm{e}^{\theta \sigma r} r^{N-1} \varphi^{\prime}+\frac{N-1}{r} \mathrm{e}^{\theta \sigma r} r^{N-1} \varphi^{\prime}+\mathrm{e}^{\theta \sigma r} r^{N-1} \varphi^{\prime \prime}=\mathrm{e}^{\theta \sigma r} r^{N-1} H \varphi,
$$

then $\mathrm{e}^{\theta \sigma r} r^{N-1} \varphi^{\prime}$ is strictly increasing on $[0, R]$. Hence, $\varphi^{\prime}(R)>\varphi^{\prime}(0)=0$, a contradiction. Since $\varphi^{\prime}$ is positive, we have $\varphi^{\prime \prime} \leq H \varphi$. Then using the initial conditions and comparison arguments, $\varphi(r) \leq \lambda \mathrm{e}^{r \sqrt{H}}$ for $r \in[0, \infty)$, and thus (2) follows. Finally, we define $\bar{\varphi}(r)=\mathrm{e}^{\theta \sigma r} r^{N-1} \varphi(r)$. Then $\bar{\varphi}\left(r_{0}\right) \geq \lambda \mathrm{e}^{\theta \sigma r_{0}} r_{0}^{N-1}$ and

$$
\bar{\varphi}^{\prime}\left(r_{0}\right)=\theta \sigma \mathrm{e}^{\theta \sigma r_{0}} r_{0}^{N-1} \varphi\left(r_{0}\right)+\frac{N-1}{r_{0}} \mathrm{e}^{\theta \sigma r_{0}} r_{0}^{N-1} \varphi\left(r_{0}\right)+\mathrm{e}^{\theta \sigma r} r_{0}^{N-1} \varphi^{\prime}\left(r_{0}\right) \geq 0 .
$$

Furthermore,

$$
\begin{aligned}
\bar{\varphi}^{\prime \prime}= & 2 \theta \sigma \frac{N-1}{r} \mathrm{e}^{\theta \sigma r} r^{N-1} \varphi+2 \theta \sigma \mathrm{e}^{\theta \sigma r} r^{N-1} \varphi^{\prime}+(\theta \sigma)^{2} \mathrm{e}^{\theta \sigma r} r^{N-1} \varphi \\
& +\frac{(N-1)(N-2)}{r^{2}} \mathrm{e}^{\theta \sigma r} r^{N-1} \varphi+2 \frac{N-1}{r} \mathrm{e}^{\theta \sigma r} r^{N-1} \varphi^{\prime}+\mathrm{e}^{\theta \sigma r} r^{N-1} \varphi^{\prime \prime} \\
= & 2 \theta \sigma \frac{N-1}{r} \mathrm{e}^{\theta \sigma r} r^{N-1} \varphi+\theta \sigma \mathrm{e}^{\theta \sigma r} r^{N-1} \varphi^{\prime}+(\theta \sigma)^{2} \mathrm{e}^{\theta \sigma r} r^{N-1} \varphi \\
& +\frac{(N-1)(N-2)}{r^{2}} \mathrm{e}^{\theta \sigma r} r^{N-1} \varphi+\frac{N-1}{r} \mathrm{e}^{\theta \sigma r} r^{N-1} \varphi^{\prime}+H \bar{\varphi} \\
\geq & H \bar{\varphi},
\end{aligned}
$$

since $\varphi>0, \varphi^{\prime}>0$. Using again comparison arguments, we obtain

$$
\bar{\varphi}(r) \geq \frac{\lambda \mathrm{e}^{\theta \sigma r_{0}} r_{0}^{N-1}}{2}\left(\mathrm{e}^{\left(r-r_{0}\right) \sqrt{H}}+\mathrm{e}^{-\left(r-r_{0}\right) \sqrt{H}}\right),
$$

which gives (3).

Now let $\psi$ be the solution of

$$
\left\{\begin{array}{l}
\psi^{\prime \prime}(r)+\frac{N-1}{r} \psi^{\prime}(r)=-\theta \sigma \psi^{\prime}+H \psi, \\
\psi(2 R)=A \\
\psi^{\prime}(0)=0 .
\end{array}\right.
$$

Clearly $\psi$ satisfies the assumptions in (4) for a suitable $\lambda$, so $\psi^{\prime}>0$. Recall that $\sigma=\|m\|_{C^{1}\left(B_{2 R}\right)}$, thus we have

$$
\psi^{\prime \prime}(r)+\frac{N-1}{r} \psi^{\prime}(r) \leq-\theta \psi^{\prime} \nabla m \cdot \frac{|x|}{r}+H \psi .
$$

If we let $v(x)=\psi(|x|)$, then by construction we have that $v$ is a radially symmetric function with $-\Delta v \geq \theta \nabla m \cdot \nabla v-H v$ in $B_{2 R}, v=A$ on $\partial B_{2 R}$, and hence, by maximum principle, $0 \leq u(x) \leq v(x)$ in $B_{2 R}$. Moreover, since $\psi$ is an increasing function, if we prove that $\psi(R) \leq C(\delta, R, \sigma) A \mathrm{e}^{-\delta \sqrt{H}}$, then we will obtain the required bound for $\|u\|_{L^{\infty}\left(B_{R}\right)}$ and the proof of the lemma will be concluded. Using (3) and choosing $r_{0}=\tau R, \tau \in(0,1)$, we obtain 


$$
A=\psi(2 R) \geq \frac{\lambda \mathrm{e}^{\theta \sigma R(\tau-2)}}{2^{N}} \tau^{N-1} \mathrm{e}^{(2-\tau) R \sqrt{H}},
$$

that gives

$$
\lambda \leq A \frac{2^{N}}{\mathrm{e}^{\theta \sigma R(\tau-2)} \tau^{N-1}} \mathrm{e}^{(-2+\tau) R \sqrt{H}} .
$$

Substituting in the inequality in (2), we finally have

$$
\psi(R) \leq A \frac{2^{N}}{\mathrm{e}^{\theta \sigma R(\tau-2)} \tau^{N-1}} \mathrm{e}^{(-1+\tau) R \sqrt{H}},
$$

then setting $\delta=1-\tau$, provides the desired inequality.

\section{Asymptotic of the Blow up Sequence}

We deduce from Section 2 that the solutions of system (2) is uniform bounded in $L^{\infty}(\Omega)$. For any compact set $K \subset K^{\prime} \subset \subset \Omega$, we are aim to show that the Lipschitz semi-norm of solutions to system (2) is bounded in $K$, uniformly in $k$. To begin with, let $\eta$ be a cut-off function such that $0 \leq \eta \leq 1, \eta \equiv 1$ in $K$ and supp $\eta=: K^{\prime} \subset \subset \Omega$, we want to show that there exist a constant $C>0$ independent of $k$ such that,

$$
\max \left\{\sup _{x \in \Omega}\left|\nabla\left(\eta u_{k}\right)\right|, \sup _{x \in \Omega}\left|\nabla\left(\eta v_{k}\right)\right|\right\} \leq C,
$$

from which the desired result follows. Inspired from the work of Soave and Zilio in [30], we assume by contradiction that, up to a subsequence, it holds

$$
L_{k}:=\max \left\{\sup _{x \in \Omega}\left|\nabla\left(\eta u_{k}\right)\right|, \sup _{x \in \Omega}\left|\nabla\left(\eta v_{k}\right)\right|\right\} \rightarrow+\infty .
$$

Without loss of generality, we may assume that the supremum is achieved by $u_{k}$ at a point $x_{k} \in K^{\prime}$, that is

$$
L_{k}=\left|\nabla u_{k}\left(x_{k}\right)\right| \rightarrow+\infty .
$$

Now we introduce two blow-up sequences

$$
\begin{gathered}
w_{k}(x)=\frac{\eta\left(x_{k}\right) u_{k}\left(x_{k}+r_{k} x\right)}{L_{k} r_{k}}, \quad z_{k}(x)=\frac{\eta\left(x_{k}\right) v_{k}\left(x_{k}+r_{k} x\right)}{L_{k} r_{k}}, \quad x \in \Omega_{k}, \\
\bar{w}_{k}(x)=\frac{\left(\eta u_{k}\right)\left(x_{k}+r_{k} x\right)}{L_{k} r_{k}}, \quad \bar{z}_{k}(x)=\frac{\left(\eta v_{k}\right)\left(x_{k}+r_{k} x\right)}{L_{k} r_{k}}, \quad x \in \Omega_{k} .
\end{gathered}
$$

where $\Omega_{k}:=\frac{\Omega-x_{k}}{r_{k}}$. We choose the scaling factor $r_{k}>0$ in such a way that

$$
\begin{aligned}
& \bar{w}_{k}(0)+\bar{z}_{k}(0)=\frac{\left(\eta u_{k}\right)\left(x_{k}\right)+\left(\eta v_{k}\right)\left(x_{k}\right)}{L_{k} r_{k}}=1 \\
& \Rightarrow r_{k}=\frac{\left(\eta u_{k}\right)\left(x_{k}\right)+\left(\eta v_{k}\right)\left(x_{k}\right)}{L_{k}} \rightarrow 0 .
\end{aligned}
$$

Note that, since $K^{\prime} \subset \subset \Omega$, we have $\Omega_{k} \rightarrow \mathbb{R}^{N}$ as $k \rightarrow \infty$. Furthermore, if $\left(u_{k}, v_{k}\right)$ is a solution to $(2)$, then $\left(w_{k}, z_{k}\right)$ satisfies 


$$
\begin{cases}-\nabla \cdot\left(\nabla w_{k}-\alpha w_{k} \nabla m_{k}\right)=r_{k}^{2} w_{k} m_{k}-b M_{k} w_{k}^{2}-k M_{k} w_{k} z_{k} & \text { in } \Omega_{k}, \\ -\nabla \cdot\left(\nabla z_{k}-\beta z_{k} \nabla m_{k}\right)=r_{k}^{2} z_{k} m_{k}-c M_{k} z_{k}^{2}-k M_{k} w_{k} z_{k} & \text { in } \Omega_{k},\end{cases}
$$

where

$$
m_{k}(x)=m\left(x_{k}+r_{k} x\right) \text { and } M_{k}:=L_{k} r_{k}^{3} / \eta\left(x_{k}\right) .
$$

The following lemma focuses on some preliminary properties of the blow up sequences.

Lemma 4. In the previous blow-up setting, the following assertions hold:

1) $\nabla m_{k} \rightarrow \overrightarrow{0}, \Delta m_{k} \rightarrow 0$, uniformly in $\Omega_{k}$ as $k \rightarrow+\infty$, in particular,

$$
m_{k}(x) \rightarrow m_{0} \text { for some constant } m_{0}>0 \text {; }
$$

2) we have

$$
r_{k}^{2} w_{k} m_{k}-b M_{k} w_{k}^{2} \rightarrow 0, r_{k}^{2} z_{k} m_{k}-c M_{k} z_{k}^{2} \rightarrow 0,
$$

uniformly in all $\Omega_{k}$ as $k \rightarrow \infty$;

3) the sequence $\left\{\bar{w}_{k}\right\}$ and $\left\{\bar{z}_{k}\right\}$ have uniformly bounded Lip-seminorm:

$$
\max \left\{\sup _{x \neq y} \frac{\left|\bar{w}_{k}(x)-\bar{w}_{k}(y)\right|}{x-y}, \sup _{x \neq y} \frac{\left|\bar{z}_{k}(x)-\bar{z}_{k}(y)\right|}{x-y}\right\} \leq 1 ;
$$

furthermore $\left|\nabla \bar{w}_{k}(0)\right|=1$ and $\left|\nabla w_{k}(0)\right| \rightarrow 1$ as $k \rightarrow+\infty$;

4) there exist $w, z$, globally Lipschitz continuous in $\mathbb{R}^{N}$ with Lipschitz constant equal to 1 , such that up to a subsequence:

$$
\begin{aligned}
& w_{k} \rightarrow w, z_{k} \rightarrow z \text { in } C_{l o c}\left(\mathbb{R}^{N}\right), \\
& \bar{w}_{k} \rightarrow w, \bar{z}_{k} \rightarrow z \text { in } C_{l o c}\left(\mathbb{R}^{N}\right) ;
\end{aligned}
$$

5) there holds $w_{k} \rightarrow w, z_{k} \rightarrow z$ in $H_{\text {loc }}^{1}\left(\mathbb{R}^{N}\right)$ as $k \rightarrow \infty$, and for any $r>0$ there exist $C>0$, independent of $k$, such that

$$
\int_{B_{r}(0)} k M_{k} w_{k} z_{k} \leq C .
$$

If $k M_{k} \rightarrow \infty$, then $w_{k} z_{k} \rightarrow 0$ as $k \rightarrow \infty$. Moreover the limit $\mathrm{w}, \mathrm{z}$ satisfies

$$
\begin{cases}-\Delta w=0 & \text { in }\{w>0\}, \\ -\Delta z=0 & \text { in }\{z>0\}, \\ w z=0 & \text { in } \mathbb{R}^{N}, \\ w, z \geq 0 & \text { in } \mathbb{R}^{N} .\end{cases}
$$

Proof. 1) Since $m \in C^{2}(\bar{\Omega})$, then for every $x \in \Omega_{k}$,

$$
\begin{aligned}
& \left|\nabla m_{k}(x)\right|=r_{k}\left|\nabla m\left(x_{k}+r_{k} x\right)\right| \leq r_{k}\|m\|_{C^{1}(\bar{\Omega})} \rightarrow 0 \text { as } k \rightarrow+\infty, \\
& \left|\Delta m_{k}(x)\right|=r_{k}^{2}\left|\Delta m\left(x_{k}+r_{k} x\right)\right| \leq r_{k}^{2}\|m\|_{C^{2}(\bar{\Omega})} \rightarrow 0 \text { as } k \rightarrow+\infty .
\end{aligned}
$$

Note also that $m(x)$ is positive somewhere in $\Omega$, thus there exists a positive constant $m_{0}$, such that $m_{k} \rightarrow m_{0}$.

2) By Lemma 2 and the definitions of $w_{k}$ and $\eta$, we have 


$$
\begin{aligned}
r_{k}^{2} w_{k} m_{k}-b M_{k} w_{k}^{2} & =\frac{r_{k} \eta\left(x_{k}\right)\left(u_{k} m_{k}\right)\left(x_{k}+r_{k} x\right)}{L_{k}}-b \frac{r_{k} \eta\left(x_{k}\right) u_{k}^{2}\left(x_{k}+r_{k} x\right)}{L_{k}} \\
& \leq C \frac{r_{k}}{L_{k}} \rightarrow 0 .
\end{aligned}
$$

Similarly, we have $r_{k}^{2} z_{k} m_{k}-c M_{k} z_{k}^{2} \rightarrow 0$.

The uniform bound on the Lipschitz seminorm of $\bar{w}_{k}, \bar{z}_{k}$ and the fact that $\left|\nabla \bar{w}_{k}(0)\right|=1$, are direct consequence of the definitions. Moreover

$$
\nabla \bar{w}_{k}(0)=\frac{u_{k}\left(x_{k}\right) \nabla \eta\left(x_{k}\right)}{L_{k}}+\frac{\eta\left(x_{k}\right) \nabla u_{k}\left(x_{k}\right)}{L_{k}}=o(1)+\nabla w_{k}(0),
$$

as $k \rightarrow \infty$, then (3) holds.

4) We will only prove the estimate of $w_{k}$ and $\bar{w}_{k}$, that of $z_{k}$ and $\bar{z}_{k}$ are similar. For any fixed $r>0$, we may let $k$ sufficient large such that $B_{r}(0) \subset \subset K$. The sequence $\left\{\bar{w}_{k}\right\}$ has a uniformly Lipschitz seminorm in $\overline{B_{r}(0)}$, and is uniformly bounded in 0 . Hence by the Ascoli-Arzelà theorem, it is uniformly convergent (up to a subsequence) to some $w \in C\left(\overline{B_{r}(0)}\right)$ having Lipschitz-seminorm bounded by 1 . To complete the proof, we shall show that $w_{k}-\bar{w}_{k} \rightarrow 0$ as $k \rightarrow+\infty$ in $C_{\text {loc }}\left(\mathbb{R}^{N}\right)$. To this aim, it is sufficient to observe that for any compact $K \subset \mathbb{R}^{N}$,

$$
\begin{aligned}
\sup _{x \in K}\left|w_{k}(x)-\bar{w}_{k}(x)\right| & =\sup _{x \in K} \frac{u_{k}\left(x_{k}+r_{k} x\right)}{L_{k} r_{k}}\left|\eta\left(x_{k}\right)-\eta\left(x_{k}+r_{k} x\right)\right| \\
& \leq \sup _{x \in K} \frac{l C}{L_{k}}|x|,
\end{aligned}
$$

where $I$ denotes the Lipschitz constant of $\eta, C$ is the uniformly boundedness of $\left\{u_{k}\right\}$. Since $L_{k} \rightarrow \infty$ and $K$ is compact, the desired result follows.

5) To prove (7), it is sufficient to test the equation for $w_{k}$ against a smooth cut-off function $0 \leq \varphi \leq 1$ such that $\varphi=1$ in $B_{r}(0)$ and $\varphi=0$ in $\mathbb{R}^{N} \backslash B_{2 r}(0)$, we obtain:

$$
\int_{B_{r}(0)} k M_{k} w_{k} z_{k} \leq \int_{B_{2 r}(0)}\left|\left(r_{k}^{2} m_{k} w_{k}-b M_{k} w_{k}^{2}\right) \varphi+w_{k} \Delta \varphi\right|+\int_{B_{2 r}(0)}\left|\alpha w_{k} \nabla m_{k} \cdot \nabla \varphi\right| .
$$

By the uniform boundedness of $\left\{w_{k}\right\}$ in compact sets and the fact that $\left|\nabla m_{k}\right| \rightarrow 0$, there exists a constant $C>0$ independent of $k$, such that

$$
\int_{B_{r}(0)} k M_{k} w_{k} z_{k} \leq C .
$$

Testing the equation for $w_{k}$ against $w_{k} \varphi^{2}$, we also deduce that

$$
\begin{aligned}
& \frac{1}{4} \int_{B_{r}(0)}\left|\nabla w_{k}\right|^{2} \leq 4 \int_{B_{2 r}(0)} w_{k}^{2}\left(\varphi^{2} \alpha^{2}\left|\nabla m_{k}\right|^{2}+|\nabla \varphi|^{2}\right) \\
& +2 \int_{B_{2 r}(0)} \alpha w_{k}^{2} \varphi\left|\nabla m_{k}\right||\nabla \varphi|+\left(r_{k}^{2} m_{k} w_{k}-b M_{k} w_{k}^{2}\right) w_{k} \varphi^{2} \leq C,
\end{aligned}
$$

where $C$ is a positive constant independent of $k$. This implies that, up to a subsequence,

$$
w_{k} \rightarrow w \text { weakly in } H^{1}\left(B_{r}\right), w_{k} \rightarrow w \text { in } L^{2}\left(B_{r}\right) .
$$

To prove the strong convergence, we test the equation for $w_{k}$ against $\left(w_{k}-w\right)$, 
and recalling that $w_{k} \rightarrow w$ uniformly in $B_{r}$, we deduce that as $k \rightarrow \infty$,

$$
\begin{aligned}
& \left|\int_{B_{r}} \nabla w_{k} \cdot \nabla\left(w_{k}-w\right)\right| \\
& =\left|\int_{\partial B_{r}} \partial_{\nu} w_{k}\left(w_{k}-w\right)\right|+\mid \int_{B_{r}}\left(-\alpha \nabla m_{k} \nabla w_{k}-\alpha w_{k} \Delta m_{k}\right. \\
& \left.\quad+r_{k}^{2} m_{k} w_{k}-b M_{k} w_{k}^{2}\right)\left(w_{k}-w\right)-k M_{k} w_{k} z_{k}\left(w_{k}-w\right) \mid \\
& \leq\left\|w_{k}-w\right\|_{L^{\infty}\left(B_{r}\right)} \int_{\partial B_{r}}\left|\partial_{\nu} w_{k}\right| \\
& \quad+\left\|w_{k}-w\right\|_{L^{\infty}\left(B_{r}\right)} \int_{B_{r}}\left|-\alpha \nabla m_{k} \nabla w_{k}-\alpha w_{k} \Delta m_{k}+r_{k}^{2} m_{k} w_{k}-b M_{k} w_{k}^{2}\right| \\
& \rightarrow 0 .
\end{aligned}
$$

From this we can pass from the weak convergence to the strong one.

To prove (8), we note that

$$
\begin{aligned}
-\Delta\left(w_{k}-z_{k}\right)= & \left(-\alpha \nabla w_{k}+\beta \nabla z_{k}\right) \nabla m_{k}+\left(-\alpha w_{k}+\beta z_{k}\right) \Delta m_{k} \\
& +\left(r_{k}^{2} m_{k} w_{k}-b M_{k} w_{k}^{2}\right)-\left(r_{k}^{2} m_{k} z_{k}-c M_{k} z_{k}^{2}\right) .
\end{aligned}
$$

By strong $H^{1}$ convergence and (1), above equation can be passing to the limit. So up to a subsequence, we have in the distribute sense that

$$
-\Delta(w-z)=0 .
$$

Since $w z=0$, we have $w-z=w$ in $\{w>0\}$, and thus

$$
-\Delta w=0 \text { in }\{w>0\} \text {. }
$$

Similarly, the result holds for $z$. This completes the proof of Lemma 4 .

Lemma 5. The limit function $(w, z)$ is not constant. In particular, $w$ is neither trivial nor constant.

Proof. We divide the proof according to properties of $k M_{k}$.

Case 1. $\left(k M_{k}\right)$ is bounded. The equation for $w_{k}$ can be rewrite as:

$$
-\Delta w_{k}+\alpha \nabla w_{k} \cdot \nabla m_{k}=-\alpha w_{k} \Delta m_{k}+r_{k}^{2} m_{k} w_{k}-b M_{k} w_{k}^{2}-k M_{k} w_{k} z_{k} .
$$

Since $\left\{w_{k}\right\}$ is uniformly bounded in any compact set of $\mathbb{R}^{N}$, by standard regularity theory for elliptic equations, we deduce that for every compact $K \subset \mathbb{R}^{N}$ there exist $C>0$ independent of $k$ such that $\left\|w_{k}\right\|_{C^{1, \alpha}(K)} \leq C$. This implies that, up to a subsequence

$$
w_{k} \rightarrow w \text { in } C_{\text {loc }}^{1, \alpha}\left(\mathbb{R}^{N}\right) \text {, for any } 0<\alpha<1 .
$$

So that in particular $|\nabla w(0)|=\lim _{k \rightarrow \infty}\left|\nabla w_{k}(0)\right|=1$, and $(w, z)$ cannot be a vector of constant functions.

Case 2. $k M_{k} \rightarrow+\infty$. By Lemma 4 (5) we infer that $w z \equiv 0$ in $\mathbb{R}^{N}$, and the choice of $r_{k}$ implies that $w_{k}(0)+z_{k}(0)=1$, so there are only two possibilities: either $w(0)=0$, or $w(0)=1$.

Assume at first that $w(0)=0$, then $z(0)=1$, and by continuity of $w, z$ it results that $w \equiv 0$ in an open neighbourhood of 0 . Moreover, there exists $0<r \ll 1$, such that

$$
z_{k}(0) \geq \frac{7}{8} \text { in } B_{r}(0),
$$

for sufficient large $k$. Thanks to Lemma 4 (4), we have 


$$
\left|z_{k}(x)-z_{k}(0)\right| \leq\left|z_{k}(x)-\bar{z}_{k}(x)\right|+\left|\bar{z}_{k}(x)-\bar{z}_{k}(0)\right| \leq o(1)+|x| \leq o(1)+\frac{1}{2},
$$

as $k \rightarrow \infty$, for every $x \in B_{r / 2}(0)$. Thus, whenever $k$ is sufficiently large, $z_{k} \geq \frac{1}{8}$

in $B_{r / 2}(0)$. As a consequence, if we Let $\tilde{w}_{k}=\mathrm{e}^{-\alpha m_{k}} w_{k}$, then $\tilde{w}_{k}$ satisfies

$$
\begin{aligned}
-\Delta \tilde{w}_{k} & =\alpha \nabla \tilde{w}_{k} \cdot \nabla m_{k}+\left(r_{k}^{2} m_{k}-b M_{k} \mathrm{e}^{\alpha m_{k}} \tilde{w}_{k}\right) \tilde{w}_{k}-k M_{k} \tilde{w}_{k} z_{k} \\
& \leq \alpha \nabla \tilde{w}_{k} \cdot \nabla m_{k}+\left(r_{k}^{2} m_{k}-b M_{k} \mathrm{e}^{\alpha m_{k}} \tilde{w}_{k}-\frac{1}{8} k M_{k}\right) \tilde{w}_{k} \\
& \leq \alpha \nabla \tilde{w}_{k} \cdot \nabla m_{k}-\frac{1}{16} k M_{k} \tilde{w}_{k} .
\end{aligned}
$$

By Lemma 3,

$$
\tilde{w}_{k} \leq C^{\prime} \mathrm{e}^{-C^{\prime \prime} \sqrt{k M_{k}}} \text { in } B_{r / 4} .
$$

Hence for every $x \in B_{r / 4}(0)$,

$$
\left|-\Delta \tilde{w}_{k}-\alpha \nabla \tilde{w}_{k} \cdot \nabla m_{k}\right| \leq C .
$$

Note that $\left|\nabla m_{k}\right| \rightarrow 0$. By standard regularity theory for elliptic equations, we have $\left\|\tilde{w}_{k}\right\|_{C^{1+\alpha}\left(B_{r / 4}\right)} \leq C$. Note also that $w_{k}=\mathrm{e}^{\alpha m_{k}} \tilde{w}_{k}$ and $\left\|m_{k}\right\|_{C^{2}\left(B_{r}(0)\right)} \rightarrow 0$ (by Lemma 4), we then deduce that

$$
\left\|w_{k}\right\|_{C^{1+\alpha}} \leq C \text { in } B_{r / 4}(0) .
$$

This implies that up to a subsequence $w_{k} \rightarrow w$ in $C^{1}\left(B_{1 / 4}\right)$. In particular $|\nabla w(0)|=1$, in contradiction with the fact that $w \equiv 0$ in a neighbourhood of 0 . Thus, the case $w(0)=0$ is impossible, therefore $w(0)=1$. As a consequence the same argument described above provides $w_{k}(x) \geq \frac{1}{8}$ in $B_{r / 2}(0)$. If we let $\tilde{z}_{k}=\mathrm{e}^{-\beta m_{k}} z_{k}$, then $\tilde{z}_{k}$ satisfies

$$
\begin{cases}-\Delta \tilde{z}_{k} \leq \alpha \nabla \tilde{z}_{k} \cdot \nabla m_{k}-\frac{1}{16} k M_{k} \tilde{z}_{k} & \text { in } B_{r / 2}, \\ \tilde{z}_{k} \geq 0 & \text { in } B_{r / 2}, \\ \tilde{z}_{k} \leq A & \text { in } B_{r / 2} .\end{cases}
$$

By Lemma 3 again,

$$
\tilde{z}_{k} \leq C^{\prime} \mathrm{e}^{-C^{\prime \prime} \sqrt{k M_{k}}} \text { in } B_{r / 4}
$$

By the uniform boundedness of the sequence $\left\{w_{k}\right\}$ in $B_{1 / 4}$, we infer that,

$$
\left|-\Delta \tilde{w}_{k}-\alpha \nabla \tilde{w}_{k} \cdot \nabla m_{k}\right| \leq C \text {. }
$$

And hence up to a subsequence $w_{k} \rightarrow w$ in $C^{1}\left(B_{1 / 4}\right)$. In particular, by Lemma 4 (3) we have

$$
|\nabla w(0)|=\lim _{k}\left|\nabla w_{k}(0)\right|=1
$$

which completes the proof.

Lemma 6. There exist $C>0$ such that $k M_{k} \geq C$.

Proof. Let us assume by contradiction that there exists a subsequence $k_{n} M_{k_{n}} \rightarrow 0$. Reasoning as in the previous lemma, the limiting function $(w, z)$ 
satisfies

$$
\begin{cases}-\Delta w=0 & \text { in } \mathbb{R}^{N}, \\ -\Delta z=0 & \text { in } \mathbb{R}^{N},\end{cases}
$$

and $w, z \geq 0$, thus thanks to the Liouville theorem, $w, z$ are constant. This contradicts the fact that $|\nabla w(0)|=1$.

We conclude this section by summing up what we proved so far in the following statement.

Proposition 7. Under the previous notations, we have

1) Up to a subsequence

$$
\begin{aligned}
& w_{n} \rightarrow w, z_{n} \rightarrow z \text { in } C_{\text {loc }}\left(\mathbb{R}^{N}\right), \\
& \bar{w}_{n} \rightarrow w, \bar{z}_{n} \rightarrow z \text { in } C_{\text {loc }}\left(\mathbb{R}^{N}\right) ;
\end{aligned}
$$

$w, z$ is non-trivial and non-constant, and in particular $|\nabla w(0)|=1$;

2) There exist $C>0$ such that $k M_{k} \geq C$;

3) If $\left(k M_{k}\right)$ is bounded, then

$$
\begin{cases}-\Delta w=-M_{\infty} w z & \text { in } \mathbb{R}^{N} \\ -\Delta z=-M_{\infty} w z & \text { in } \mathbb{R}^{N} \\ w z \geq 0 & \text { in } \mathbb{R}^{N}\end{cases}
$$

where $k M_{k} \rightarrow M_{\infty}$ as $k \rightarrow \infty$;

4) If $k M_{k} \rightarrow+\infty$, then both $w$ and $z$ are subharmonic in $\mathbb{R}^{N}$, and

$$
\begin{cases}-\Delta w=0 & \text { in }\{w>0\} \\ -\Delta z=0 & \text { in }\{z>0\} \\ w z=0 & \text { in } \mathbb{R}^{N} \\ w, z \geq 0 & \text { in } \mathbb{R}^{N} .\end{cases}
$$

\section{Uniform Lipschitz Bounds with Respect to $k$}

This section is devoted to the study of the Lipschitz uniform continuty of the system (2). In Section 3, we have proved that the limit $(w, z)$ is non-trivial and non-constant, and in particular $|\nabla w(0)|=1$ (Proposition 7). In what follows, we will show that one of the components of $(w, z)$ is identically zero and the other is a constant, which bring us to a contradiction.

For any given $u, v \in H_{\text {loc }}^{1}\left(\mathbb{R}^{N}\right)$ functions, we let

$$
\Phi(r):=\frac{1}{r^{4}} \int_{B_{r}} \frac{|\nabla u|^{2}}{|x|^{n-2}} \mathrm{~d} x \int_{B_{r}} \frac{|\nabla v|^{2}}{|x|^{n-2}} \mathrm{~d} x .
$$

we shall make use of the celebrated almost monotonicity formula of Callarelli-Jerison-Kening, which we recall here in its original formulation.

Theorem 8. (Callarelli-Jerison-Kening almost monotonicity). Suppose $u, v$ are non-negative, continuous functions on the unit ball $B_{1}$. Suppose that $-\Delta u \leq 1$ and $-\Delta v \leq 1$ in the sense of distributions and that $u(x) v(x)=0$ for all $x \in B_{1}$. Then there exist a constant $C$ depending only on dimension such that 
for every $0<r \leq 1$ :

$$
\Phi(r) \leq C\left(1+\int_{B_{r}} \frac{|\nabla u|^{2}}{|x|^{n-2}}+\int_{B_{r}} \frac{|\nabla v|^{2}}{|x|^{n-2}}\right)
$$

Moreover, if $u$ and $v$ satisfy the same assumptions also in the ball $B_{2}$, then there exist a dimensional constant $C>0$ such that

$$
\Phi(r) \leq C\left(1+\int_{B_{2}} u^{2}+\int_{B_{2}} v^{2}\right)^{2}, 0<r \leq 1 .
$$

Now we consider the following systems

$$
\begin{cases}-\nabla \cdot\left(\nabla w_{k}-\alpha w_{k} \nabla m_{k}\right)=r_{k}^{2} w_{k} m_{k}-b M_{k} w_{k}^{2}-k M_{k} w_{k} z_{k} & \text { in } \Omega_{k}, \\ -\nabla \cdot\left(\nabla z_{k}-\beta z_{k} \nabla m_{k}\right)=r_{k}^{2} z_{k} m_{k}-c M_{k} z_{k}^{2}-k M_{k} w_{k} z_{k} & \text { in } \Omega_{k} .\end{cases}
$$

Therefore,

$$
\begin{aligned}
-\Delta\left(w_{k}-z_{k}\right)= & \left(-\alpha \nabla w_{k}+\beta \nabla z_{k}\right) \nabla m_{k}+\left(-\alpha w_{k}+\beta z_{k}\right) \Delta m_{k} \\
& +\left(r_{k}^{2} m_{k} w_{k}-b M_{k} w_{k}^{2}\right)-\left(r_{k}^{2} m_{k} z_{k}-c M_{k} z_{k}^{2}\right) .
\end{aligned}
$$

Notice that $r_{k}^{2} m_{k} w_{k}-b M_{k} w_{k}^{2} \rightarrow 0, r_{k}^{2} m_{k} z_{k}-c M_{k} z_{k}^{2} \rightarrow 0, m_{k} \rightarrow m_{0}$. Hence in the sense of distributions that $-\Delta\left(w_{k}-z_{k}\right) \rightarrow 0$, and in particular

$$
\left\{\begin{array}{l}
-\Delta\left(w_{k}-z_{k}\right)^{+} \leq 1 \\
-\Delta\left(w_{k}-z_{k}\right)^{-} \leq 1
\end{array}\right.
$$

for $k$ sufficiently large.

Lemma 9. There exist a constant $C>0$ independent of $k$ such that for any $r \in(0,+\infty)$ and $x_{0} \in \Omega_{k}$,

$$
\frac{1}{r^{N}} \int_{B_{r}\left(x_{0}\right)}\left|\nabla\left(w_{k}-z_{k}\right)^{+}\right|^{2} \cdot \frac{1}{r^{N}} \int_{B_{r}\left(x_{0}\right)}\left|\nabla\left(w_{k}-z_{k}\right)^{-}\right|^{2} \leq C .
$$

Proof. By (9), it follows that the positive and negative part of $\left(w_{k}-z_{k}\right)$ fall under the assumptions of Theorem 8 , and in particular

$$
\begin{aligned}
& \frac{1}{r^{2 N}} \int_{B_{r}\left(x_{0}\right)}\left|\nabla\left(w_{k}-z_{k}\right)^{+}\right|^{2} \int_{B_{r}\left(x_{0}\right)}\left|\nabla\left(w_{k}-z_{k}\right)^{-}\right|^{2} \\
& \leq \frac{1}{r^{4}} \int_{B_{r}\left(x_{0}\right)} \frac{\left|\nabla\left(w_{k}-z_{k}\right)^{+}\right|^{2}}{\left|x-x_{0}\right|^{N-2}} \cdot \int_{B_{r}\left(x_{0}\right)} \frac{\left|\nabla\left(w_{k}-z_{k}\right)^{-}\right|^{2}}{\left|x-x_{0}\right|^{N-2}} \\
& \leq C\left(1+\int_{B_{r}\left(x_{0}\right)} w_{k}^{2}+z_{k}^{2}\right)^{2} \leq C,
\end{aligned}
$$

where $C>0$ is independent of $k$.

Corollary 1. Any blow-up limit $(w, z)$ is made of ordered functions, that is if

$$
w_{k} \rightarrow w, z_{k} \rightarrow z
$$

then either $w \leq z$ or $z \leq w$, in $C_{\text {loc }}\left(\mathbb{R}^{N}\right)$.

Proof. Indeed, scaling properly of the estimate (10), we obtain for every $r \in\left(0,1 / r_{k}\right)$ and $k$ large enough 


$$
\frac{1}{r^{N}} \int_{B_{r}\left(x_{0}\right)}\left|\nabla\left(w_{k}-z_{k}\right)^{+}\right|^{2} \cdot \frac{1}{r^{N}} \int_{B_{r}\left(x_{0}\right)}\left|\nabla\left(w_{k}-z_{k}\right)^{-}\right|^{2} \leq \frac{\eta\left(x_{k}\right)^{4}}{L_{k}^{4}} \rightarrow 0
$$

as $k \rightarrow+\infty$. The conclusion follows by strong $H_{l o c}^{1}\left(\mathbb{R}^{N}\right)$ convergence of the blow-up sequence and by the continuity of the blow-up limit.

In order to complete the proof of Theorem 1, we need the following classical result, for which we refer to Lemma 2 in [31].

Lemma 10. Let $1<p<\infty$, and let $u \in L_{\text {loc }}^{p}\left(\mathbb{R}^{N}\right)$ be a solution of

$$
-\Delta u \leq-|u|^{p-1} u \text { in } \mathbb{R}^{N},
$$

if we assume $u$ to be non-negative, then $u \equiv 0$.

With the lemmas above, we can now complete the proof of uniform Lipschitz bounds.

Proof of Theorem 1. According to $k M_{k}$, we divided the proof in two steps.

Step 1. The case $\left(k M_{k}\right)$ bounded. In this case by Proposition 7 the limiting function $(w, z)$ is a non-negative, non-trivial, non-constant and sublinear solution of

$$
-\Delta w=-M_{\infty} w z, \quad-\Delta z=-M_{\infty} w z .
$$

By Corollary 1 , we evince that either $w \leq z$ in $\mathbb{R}^{N}$, or $w \geq z$ in $\mathbb{R}^{N}$. Without loss of generality, we suppose that $w \neq 0$ and $w \geq z$. Thus

$$
-\Delta z=-M_{\infty} w z \leq-M_{\infty} z^{2} .
$$

Thanks to Lemma 10, we have $z \equiv 0$. But then

$$
-\Delta w=-M_{\infty} w Z \equiv 0 .
$$

Then by the classical Liouville theorem, we have $w$ is a constant, which is in contradiction with the fact that $w$ is non-trivial and non-constant.

Step 2. the case $k M_{k} \rightarrow+\infty$. In such a situation, $w z \equiv 0$. Notice that

$$
\begin{aligned}
-\Delta\left(w_{k}-z_{k}\right)= & \left(-\alpha \nabla w_{k}+\beta \nabla z_{k}\right) \nabla m_{k}+\left(-\alpha w_{k}+\beta z_{k}\right) \Delta m_{k} \\
& +\left(r_{k}^{2} m_{k} w_{k}-b M_{k} w_{k}^{2}\right)-\left(r_{k}^{2} m_{k} z_{k}-c M_{k} z_{k}^{2}\right) \rightarrow 0 .
\end{aligned}
$$

that is $-\Delta(w-z)=0$. Then Corollary 1 implies that either $w \leq z$, or $w \geq z$. Without loss of generality, we suppose that $w \geq Z$, then the classical Liouville theorem shows that

$$
w-z \equiv C \geq 0 .
$$

since $w \cdot z=0$, Therefore $(z+C) z=0$.

We deduce that $z=0$, and $w \equiv C$, this implies that $w$ is a constant, similarly, a contradiction. This completes the proof of Theorem 1 .

\section{Conclusion and Further Works}

The study of the asymptotic behavior of singular perturbed equations and systems of elliptic or parabolic type is very broad and active subject of research. In this paper, we study a competition-diffusion-advection system for two competing species in a spatially heterogenous environment. We prove the uniform Lip- 
schitz bound for solutions of the system, which extends known quasi-optimal results and covers the optimal case for this problem. We remark that the existence of uniform Lipschitz bounds is relevant not only for a pure mathematical flavour. As already observed in [29], it is necessary to obtain, rigorous qualitative description of phase separation phenomena (the uniform Hölder bounds would not be sufficient for this purpose.)

Finally, we mention that there are many interesting problems for further study. Note that we established uniform Lipschitz bound for solutions to elliptic system (2), naturally to ask whether our results can be extended to the parabolic system (1)? Up to our knowledge, the optimal Lipschitz bound for parabolic setting is unknown even for the case when $\alpha=\beta=0$ (without advection terms) in system (1). Moreover, in system (2) the advection rates $\alpha$ and $\beta$ are fixed nonzero constants, what happens if $\alpha$ and $\beta$ are $k$-dependent and are suitably large? In such situation, the regularity of the solutions remains a challenge, and it will be the object of a forthcoming paper.

\section{Acknowledgements}

We thank the Editor and the referee for their comments. The work is partially supported by PRC grant NSFC 11601224.

\section{Conflicts of Interest}

The authors declare no conflicts of interest regarding the publication of this paper.

\section{References}

[1] Chen, X.F., Hambrock, R. and Lou, Y. (2008) Evolution of Conditional Dispersal: A Reaction-Diffusion-Advection Model. Journal of Mathematical Biology, 57, 361-386. https://doi.org/10.1007/s00285-008-0166-2

[2] Cantrell, R.S. and Cosner, C. (2003) Spatial Ecology via Reaction-Diffusion Equations. Series in Mathematical and Computational Biology, John Wiley and Sons, Chichester. https://doi.org/10.1002/0470871296

[3] Cantrell, R.S., Cosner, C. and Lou, Y. (2007) Advection Mediated Coexistence of Competing Species. Proceedings of the Royal Society of Edinburgh Section A, 137, 497-518. https://doi.org/10.1017/S0308210506000047

[4] Cantrell, R.S., Cosner, C. and Lou, Y. (2006) Movement towards Better Environments and the Evolution of Rapid Diffusion. Mathematical Biosciences, 204, 199-214. https://doi.org/10.1016/j.mbs.2006.09.003

[5] Chen, X.F. and Lou, Y. (2008) Principal Eigenvalue and Eigenfunction of Elliptic Operator with Large Convection and Its Application to a Competition Model. Indiana University Mathematics Journal, 57, 627-658. https://doi.org/10.1512/iumj.2008.57.3204

[6] Zhang, S., Zhou, L. and Liu, Z. (2013) The Spatial Behavior of a Competition-Advection-Diffusion System with Strong Competition. Nonlinear Analysis: RWA, 14, 976-989. https://doi.org/10.1016/j.nonrwa.2012.08.011

[7] Crooks, E.C.M. and Dancer, E.N. (2010) Highly Nonlinear Large-Competition Lim- 
its of Elliptic Systems. Nonlinear Analysis, 73, 1447-1457.

https://doi.org/10.1016/j.na.2010.05.008

[8] Crooks, E.C.M., Dancer, E.N. and Hilhorst, D. (2007) On Long-Time Dynamics for Competition-Diffusion Systems with Inhomogeneous Dirichlet Boundary Conditions. Topological Methods in Nonlinear Analysis, 30, 1-36.

[9] Crooks, E.C.M., Dancer, E.N., Hilhorst, D., Mimura, M. and Ninomiya, H. (2004) Spatial Segregation Limit of a Competition Diffusion System with Dirichlet Boundary Conditions. Nonlinear Analysis. Real World Applications, 5, 645-665. https://doi.org/10.1016/j.nonrwa.2004.01.004

[10] Conti, M., Terracini, S. and Verzini, G. (2005) A Variational Problem for the Spatial Segregation of Reaction Diffusion Systems. Indiana University Mathematics Journal, 54, 779-815. https://doi.org/10.1512/iumj.2005.54.2506

[11] Conti, M., Terracini, S. and Verzini, G. (2005) Asymptotic Estimates for the Spatial Segregation of Competitive Systems. Advances in Mathematics, 195, 524-560. https://doi.org/10.1016/j.aim.2004.08.006

[12] Dancer, E.N. and Du, Y.H. (1994) Competing Species Equations with Diffusion, Large Interactions, and Jumping Nonlinearities. Journal of Differential Equations, 114, 434-475. https://doi.org/10.1006/jdeq.1994.1156

[13] Dancer, E.N. and Zhang, Z. (2002) Dynamics of Lotka-Volterra Competition Systems with Large Interactions. Journal of Differential Equations, 182, 470-489. https://doi.org/10.1006/jdeq.2001.4102

[14] Wang, K. and Zhang, Z. (2010) Some New Results in Competing Systems with Many Species. Annales de I Institut Henri Poincaré, 27, 739-761.

https://doi.org/10.1016/j.anihpc.2009.11.004

[15] Caffarelli, L.A., Karakhanyan, A.L. and Lin, F. (2009) The Geometry of Solutions to a Segregation Problem for Non-Divergence Systems. Journal of Fixed Point Theory and Applications, 5, 319-351. https://doi.org/10.1007/s11784-009-0110-0

[16] Caffarelli, L.A. and Lin, F. (2008) Singularly Perturbed Elliptic Systems and Multi-Valued Harmonic Functions with Free Boundaries. Journal of the American Mathematical Society, 21, 847-862. https://doi.org/10.1090/S0894-0347-08-00593-6

[17] Chang, S.M., Lin, C.S., Lin, T.C. and Lin, W.W. (2004) Segregated Nodal Domains of Two-Dimensional Multispecies Bose-Einstein Condensates. Physics D, 196, 341-361. https://doi.org/10.1016/j.physd.2004.06.002

[18] Dancer, E.N., Wang, K. and Zhang, Z. (2011) Uniform Hölder Estimate for Singulary Perturbed Parabolic Systems of Bose-Einstein Condensates and Competing Species. Journal of Differential Equations, 251, 2737-2769.

https://doi.org/10.1016/j.jde.2011.06.015

[19] Dancer, E.N., Wang, K. and Zhang, Z. (2012) The Limit Equation for the Gross-Pitaevskii Equations and S. Terracini's Conjecture. Journal of Functional Analysis, 262, 1087-1131. https://doi.org/10.1016/j.jfa.2011.10.013

[20] Tavares, H. and Terracini, S. (2012) Regularity of the Nodal Set of the Segregated Critical Configuration under a Weak Reflection Law, Calc. Var. Partial Differential Equations, 45, 273-317. https://doi.org/10.1007/s00526-011-0458-Z

[21] Wei, J. and Weth, T. (2008) Asymptotic Behaviour of Solutions of Planar Elliptic Systems with Strong Competition. Nonlinearity, 21, 305-317. https://doi.org/10.1088/0951-7715/21/2/006

[22] Liu, Z. (2009) Phase Separation of Two Component Bose-Einstein Condensates. Journal of Mathematical Physics, 50, Article ID: 102104. 
https://doi.org/10.1063/1.3243875

[23] Liu, Z. (2011) The Spatial Behavior of Rotating Two-Component Bose-Einstein Condensates. Journal of Functional Analysis, 261, 1711-1751. https://doi.org/10.1016/j.jfa.2011.05.017

[24] Zhang, S. and Liu, Z. (2015) Singularities of the Nodal Set of Segregated Configurations, Calc. Var. Partial Differential Equations, 54, 2017-2037. https://doi.org/10.1007/s00526-015-0854-x

[25] Noris, B., Tavares, H., Terracini, S. and Verzini, G. (2010) Uniform Hölder Bounds for Nonlinear Schrödinger Systems with Strong Competition. Communications on Pure and Applied Mathematics, 63, 267-302. https://doi.org/10.1002/cpa.20309

[26] Terracini, S., Verzini, G. and Zilio, A. (2016) Uniform Hölder Bounds for Strongly Sompeting Systems Involving the Square Root of the Laplacian. Journal of the $\mathrm{Eu}$ ropean Mathematical Society, 18, 2865-2924. https://doi.org/10.4171/JEMS/656

[27] Terracini, S., Verzini, G. and Zilio, A. (2014) Uniform Hölder Regularity with Small Exponent in Competing Fractional Diffusion Systems. Discrete and Continuous Dynamical Systems, 34, 2669-2691. https://doi.org/10.3934/dcds.2014.34.2669

[28] Verzini, G. and Zilio, A. (2014) Strong Competition versus Fractional Diffusion: The Case of Lotka-Volterra Interaction. Communications in Partial Differential Equations, 39, 2284-2313. https://doi.org/10.1080/03605302.2014.890627

[29] Berestycki, H., Lin, T.C., Wei, J. and Zhao, C. (2013) On Phase-Separation Models: Asymptotics and Qualitative Properties. Archive for Rational Mechanics and Analysis, 208, 163-200. https://doi.org/10.1007/s00205-012-0595-3

[30] Soave, N. and Zilio, A. (2015) Uniform Boundes for Strongly Competing Systems: The Optimal Lipschitz Case. Archive for Rational Mechanics and Analysis, 218, 647-697. https://doi.org/10.1007/s00205-015-0867-9

[31] Brezis, H. (1984) Semilinear Equation in $\mathbb{R}^{N}$ without Condition at Infinity. Applied Mathematics \& Optimization, 12, 271-282. https://doi.org/10.1007/BF01449045 